Mens

Revue d'histoire intellectuelle et culturelle

mens

\title{
La représentation sociale de l'Afrique dans le discours missionnaire canadien-français (1900-1968)
}

\section{Éric Desautels}

Volume 13, numéro 1, automne 2012

S'approprier le passé des autres : les usages de l'histoire internationale au Québec avant la Révolution tranquille

URI : https://id.erudit.org/iderudit/1019699ar

DOI : https://doi.org/10.7202/1019699ar

Aller au sommaire du numéro

Éditeur(s)

Centre de recherche en civilisation canadienne-française

ISSN

1492-8647 (imprimé)

1927-9299 (numérique)

Découvrir la revue

Citer cet article

Desautels, É. (2012). La représentation sociale de l'Afrique dans le discours missionnaire canadien-français (1900-1968). Mens, 13(1), 81-107.

https://doi.org/10.7202/1019699ar

\section{Résumé de l'article}

Par leurs récits, les missionnaires catholiques canadiens-français envoyés en Afrique ont transmis une représentation sociale de l'Afrique dès le tournant du $\mathrm{xx}^{\mathrm{e}}$ siècle. Si cette représentation était initialement basée sur une interprétation culturelle occidentale des croyances et des rites africains, elle a progressivement évolué jusque dans les années 1960. En parallèle aux conflits mondiaux et au développement d'une sphère internationale, les tensions liées à la colonisation et à la décolonisation ont joué un rôle important dans l'évolution du discours missionnaire sur l'Afrique. Les mouvements nationalistes africains et québécois ont transformé les fondements de la vocation missionnaire et du rapport à l'autre. D'une représentation basée sur l'exotisme et l'inconnu avant la Révolution tranquille, l'imaginaire collectif québécois sur l'Afrique est graduellement passé à une représentation caractérisée par la pauvreté, la souffrance et la misère. 


\title{
La représentation sociale de l'Afrique dans le discours missionnaire canadien-français (1900-1968)
}

\author{
Éric Desautels \\ Université Concordia
}

\section{Résumé}

Par leurs récits, les missionnaires catholiques canadiens-français envoyés en Afrique ont transmis une représentation sociale de l'Afrique dès le tournant du $\mathrm{Xx}^{\mathrm{e}}$ siècle. Si cette représentation était initialement basée sur une interprétation culturelle occidentale des croyances et des rites africains, elle a progressivement évolué jusque dans les années 1960. En parallèle aux conflits mondiaux et au développement d'une sphère internationale, les tensions liées à la colonisation et à la décolonisation ont joué un rôle important dans l'évolution du discours missionnaire sur l'Afrique. Les mouvements nationalistes africains et québécois ont transformé les fondements de la vocation missionnaire et du rapport à l'autre. D'une représentation basée sur l'exotisme et l'inconnu avant la Révolution tranquille, l'imaginaire collectif québécois sur l'Afrique est graduellement passé à une représentation caractérisée par la pauvreté, la souffrance et la misère.

\section{Abstract}

Through their narratives, French Canadian catholic missionaries sent to Africa constructed a social representation of Africa as soon as the beginning of the twentieth century. If this representation was at first based on a Western cultural interpretation of African rituals and beliefs, it involved considerably during the decades leading to the 1960s. In parallel with 
international conflicts and the development of globalization, tensions linked to the colonization and decolonization of Africa played an important role in the evolution of missionary discourses on Africa. What's more, nationalist movements in Africa and Quebec transformed the meaning of missionary work and of the relationship to the other. In Quebec, the image of Africa in the collective imagination went from a representation based on the exoticism of the unknown to one characterised by poverty, suffering and misery.

Dans son célèbre ouvrage Orientalism publié en 1978, Edward Said décrit l'orientalisme comme un "style de pensée basé sur une distinction ontologique et épistémologique érigée entre "l'Orient" et (la plupart du temps) "l'Occident" "1. À travers la notion d'archétype, Said décrit comment le discours sur l'Orient est structuré par une dualité culturelle. Cette dualité se traduit en un rapport entre une perspective occidentale, souvent prépondérante et positive, et la culture de l'autre, vue plutôt négativement. À partir de connaissances transmises historiquement, le regard porté sur l'Orient par les acteurs sociaux occidentaux traduirait ainsi ce type de rapport à l'autre et les représentations sociales qui en découlent. Cette forme d'interprétation, qu'elle soit erronée ou véridique, basée sur des préjugés ou non, peut mener, lorsqu'elle se cristallise dans l'imaginaire social, à des mythes collectifs. Ces interprétations peuvent aussi mener à des positions contradictoires et à des confrontations culturelles. C'est le cas de la décolonisation, qui a redéfini l'image de l'Afrique dans l'imaginaire collectif de nombreuses sociétés à travers le monde en opposant la culture coloniale à une culture de résistance ${ }^{2}$.

${ }^{1}$ Edward Said, Orientalism, London, Penguin Books, 1978 [2003], p. 2. (Nous traduisons.)

2 Jan Nederveen Pieterse et Bhikhu Parekh, «Shifting Imaginaries: Decolonization, Internal Decolonization, Postcoloniality ", dans Jan Nederveen Pieterse et Bhikhu Parekh (dir.), The Decolonization of Imagination: Culture, Knowledge and Power, Londres, Zed Books, 1995, p. 11. 
Le type d'approche proposé par Said ${ }^{3}$ apporte un éclairage intéressant afin de saisir et d'expliquer les rapports entre missionnaires et missionnés dans la première partie du xx siècle. Comme un verre grossissant, l'utilisation d'une telle perspective permet de mieux comprendre l'évolution du discours en regard des relations qui, à la fois, unissent et opposent colonisateurs et colonisés, missionnaires et missionnés. Par leurs connaissances et leurs savoirs, les missionnaires canadiens-français ont en effet transmis une représentation de l'Afrique ancrée dans l'orientalisme du début du xxe siècle. Ces rapports culturels se sont traduits, dans le champ missionnaire, par des rencontres et des échanges contrastés entre missionnaires et missionnés 4 . D'une représentation basée sur l'exotisme et l'inconnu avant la Révolution tranquille, l'imaginaire collectif québécois sur l'Afrique est graduellement passé à une représentation caractérisée par la pauvreté et la misère. Ce passage d'une forme de représentation à l'autre a été facilité, comme nous le verrons, par les tensions issues de l'impérialisme colonial et des mouvements nationalistes africains et québécois.

À l'instar de récents travaux européens sur l'influence des missions protestantes ${ }^{5}$ et sur le rôle de la religion catholique et des mouvements

3 D'autres auteurs ont développé une approche cherchant à démasquer les rapports culturels hégémoniques entre colonisateurs et colonisés. Parmi eux, notons Albert Memmi (Portrait du colonisé, précédé de Portrait du colonisateur, Paris, J.-J. Pauvert, 1966; L’homme dominé : le Noir, le colonisé, le Juif, le prolétaire, la fermme, le domestique, Paris, Gallimard, [1968] 2010), Frantz Fanon (Les damnés de la terre, Paris, La Découverte, [1961] 2002; Pour la révolution africaine : écrits politiques, Paris, La Découverte, [1952-1961] 2001), William Edward Burghardt DuBois (Africa, its Geography, People, and Products, and Africa its Place in Modern History, Millwood, KTO Press, [1930] 1977) ou encore Jacques Derrida (Le monolinguisme de l'autre ou La prothèse d'origine, Paris, Galilée, 1996).

${ }^{4}$ Sur les échanges interculturels et la transmission des idées, traditions et cultures dans l'espace missionnaire, se référer à Gilles Routhier et Frédéric Laugrand (dir.), L'espace missionnaire : lieu d'innovations et de rencontres interculturelles, Paris, Karthala; Québec, Les Presses de l'Université Laval, 2002.

5 Frédéric Fabre, Protestantisme et colonisation : l'évolution du discours de la mission protestante française au XXe siècle, Paris, Karthala, 2011; voir aussi les travaux du Oxford Centre for Mission Studies. 
jeunesse face à la décolonisation ${ }^{6}$, cet article porte sur les effets de la colonisation et de la décolonisation sur les missions canadiennesfrançaises entre 1900 et 1968. La transformation de la représentation sociale de l'Afrique dans le discours missionnaire sera mise en parallèle avec la mutation des modes d'engagement missionnaire ${ }^{7}$ : d'une mission civilisatrice, inspirée par des volontés de conversion et d'évangélisation, on passe à une conception de la vocation missionnaire étroitement liée au modèle coopératif et humanitaire. C'est ainsi que quatre périodes typiques seront explorées en fonction de l'évolution du missionnariat canadien-français : l'organisation (1900-1929), l'expansion (1929-1948), la stabilisation (1948-1958) et l'apogée $(1958-1968)^{8}$.

${ }^{6}$ Dominique Borne et Benoît Falaize (dir.), Religions et colonisation: Afrique, Asie, Océanie, Amériques, $X V I^{e}-X X^{e}$ siècle, Paris, Éditions de l'Atelier et Éditions ouvrières, 2009; Charles-Édouard Harang, Quand les jeunes catholiques découvrent le monde, Paris, Éditions du Cerf, 2010 ; Paul Coulon (dir.), Action française, décolonisation, $M^{g r}$ Lefebvre : les Spiritains et quelques crises $d u X X^{e}$ siècle, Paris, Karthala, 2009; Rebecca Rogers, "Éducation, religion et colonisation en Afrique aux XIX et $\mathrm{XX}^{\mathrm{e}}$ siècles ", CLIO Histoire, femmes et sociétés, nº 6 (1997), p. 189-194. Voir également les travaux de l'historien français Claude Prudhomme sur les missions catholiques et protestantes.

7 La perspective inverse a déjà été explorée par Lucien Laverdière, qui s'intéressait à la représentation sociale du missionnaire dans la littérature africaine : L’Africain et le missionnaire : l'image du missionnaire dans la littérature africaine d'expression française, Montréal, Bellarmin, 1987.

8 Ces périodes reposent sur un découpage typologique développé par Raymond Hostie et repris par l'historienne Micheline Dumont dans son analyse de l'évolution des congrégations religieuses féminines au $\mathrm{xx}^{\mathrm{e}}$ siècle. À l'instar de Dumont, il est préférable de délaisser la cinquième phase, soit l'extinction, qui réfere davantage aux théories classiques de la sécularisation. Selon Hostie, "le $\mathrm{xx}^{\mathrm{e}}$ siècle aurait stigmatisé la phase de stabilisation qui s'amorçait alors par trois phénomènes conjoints : la croissance numérique des effectifs, la stabilisation des structurations organisationnelles et enfin l'immobilisme des aspirations " (Micheline Dumont, "Les charismes perdus : l'avenir des congrégations religieuses féminines en l'an 2000 ", Recherches féministes, vol. 3, n 2 (1990), p. 74). Voir aussi Micheline Dumont, Les religieuses sont-elles féministes?, Montréal, Éditions Fides, 1995. 


\section{La colonisation et l'organisation des missions (1900-1929) ${ }^{9}$}

Au tournant du $\mathrm{xx}^{\mathrm{e}}$ siècle, peu de congrégations missionnaires canadiennes-françaises sont actives à l'étranger (leurs énergies se déploient principalement dans le nord et l'ouest du Canada, qui demeurent des territoires relevant de la Propagande). Les congrégations des Sœurs de Notre-Dame de la Charité du Bon Pasteur d'Angers ainsi que plusieurs Sœurs de la Providence de Montréal représentent une minorité de congrégations missionnaires à travailler et à s'organiser à l'étranger, surtout en Amérique du Sud ${ }^{10}$. Selon les historiens Jean Hamelin et Nicole Gagnon, "l'émergence d'un authentique esprit missionnaire au sein de la population canadienne-française coïncide avec la Première Guerre mondiale ${ }^{11}$ ». Les congrégations canadiennesfrançaises qui consacrent leurs activités en pays étrangers se multiplient avec la publication, en 1919 par Benoît XV, de l'encyclique Maximum Illud. Entre 1900 et 1929, de nombreuses congrégations missionnaires sont fondées au Canada français ${ }^{12}$ et les œuvres missionnaires canadiennes-françaises accroissent leurs ressources pécuniaires ${ }^{13}$. Les

9 Cet article se base essentiellement sur le discours véhiculé par les missionnaires canadiens-français dans les revues officielles de leurs congrégations. Les revues Missions d'Afrique des Pères blancs, Sxurs blanches et Annales de la Propagation de la Foi sont utilisées afin d'exposer l'évolution du discours idéologique sur l'Afrique. L'ajout d'encycliques, d'articles de journaux et de sources secondaires permet de compléter la représentation selon différents niveaux de la hiérarchie ecclésiale.

${ }^{10}$ Jean Hamelin et Nicole Gagnon, Histoire du catholicisme québécois, vol. III : Le XXe siècle, t. 1 : 1898-1940, Montréal, Éditions du Boréal Express, 1984, p. 157.

${ }^{11}$ Ibid., p. 170.

12 Voici quelques-unes des congrégations fondées ou ayant obtenu leur indépendance entre 1900 et 1930, qui se tournent vers l'Afrique : Pères blancs d'Afrique (1901), sœurs Missionnaires de Notre-Dame d'Afrique (1903), Frères Prêcheurs Dominicains (1911), Les Spiritains (1924), Frères de l'instruction chrétienne (1926), Sœurs du Christ-Roi (1928), Sœurs de l'Espérance (1928), Frères du Sacré-Cœur (1928). C’est sans compter la Compagnie de Jésus et les communautés oblates déjà présentes au Canada français et qui se tournent vers l'Afrique autour des années 1920 et 1930 .

13 L'Euvre de la Propagation de la Foi, l'CEuvre de Saint-Pierre-Apôtre, l'Euvre de la Sainte-Enfance et l'Union missionnaire du clergé sont les principales œuvres missionnaires. 
effectifs missionnaires dépassent, en 1931, 1103 missionnaires canadiensfrançais à travers le monde ${ }^{14}$.

Le tout premier missionnaire québécois arrive en Afrique en 1860, inaugurant par sa présence le début de l'activité missionnaire canadienne-française sur ce continent ${ }^{15}$. C'est toutefois le père Arthur Bouchard qui publie les premiers écrits missionnaires sur l'Afrique dans les revues canadiennes-françaises, notamment dans la version française des Annales de la Propagation de la Foi à partir de 1880. Il atteint d'ailleurs une certaine notoriété dans les cercles catholiques de l'époque. Inspiré par le récit de martyrs missionnaires, le père montréalais John Forbes effectue, de son côté, plusieurs voyages en Afrique et au Moyen-Orient dès 1886. À son retour en 1901, il fonde un postulat de la congrégation des Pères blancs d'Afrique à Québec, ce qui marque le début véritable du recrutement missionnaire africain au Canada français.

À cette époque, la représentation de l'Afrique demeure basée sur des archétypes érigés à partir de romans, d'ouvrages généraux ou de récits publiés dans les journaux de la province. Des écrits missionnaires provenant de l'Afrique se retrouvent ainsi dans des quotidiens comme Le Devoir et L'Action catholique. La revue Missions d'Afrique des Pères blancs devient, à partir de 1904, le premier moyen de grande envergure utilisé pour raconter les exploits des missionnaires en Afrique. Quatre ans plus tard, les sœurs Missionnaires de Notre-Dame d'Afrique (Sœurs blanches) lancent leur propre revue.

Les missionnaires sont souvent dépaysés à leur arrivée en terre africaine. Parler de choc culturel pour certains ne serait pas un euphémisme. Peu importe la congrégation, des thèmes comme le climat, la nature, la faune, la flore, les habitations et même la nourriture imprègnent le discours missionnaire. Ce regard extérieur sur l'Afrique permet de contribuer à une représentation sociale caractérisée

14 Hamelin et Gagnon, Histoire du catholicisme québécois, p. 157.

15 Jean-Paul Pelletier, "Les relations entre le Québec et l'Afrique 1880-1905 ", Revue canadienne des études africaines, vol. 15, n 1 (1981), p. 117. 
par l'exotisme et l'inconnu. Les récits des Sours blanches, par exemple, prennent des accents quasi anthropologiques, comme en fait foi une série d'articles parue entre 1912 et 1915 intitulée "Étude de Mœurs Africaines ", qui s'intéresse aux mœurs des populations du Soudan, de l'Ouganda et du Kenya, entre autres.

Cependant, à peu près toujours, la culture occidentale, et non seulement chrétienne, demeure un point de référence pour juger des connaissances et des croyances des Africains. Ces derniers seraient, au dire des missionnaires canadiens-français, peu " éclairés » et peu ouverts d'esprit, surtout face à la religion et à la spiritualité. Par exemple, l'oblat Odilon Chevrier, décrivant la population du Basutoland dans Le Devoir, évoque l'état " primitif " de cette contrée africaine : "Quand on parle des Basutos, il ne faut pas employer le mot de science que par euphémisme, car ils ne connaissent et ne possèdent rien qui mérite ce nom. Ils ne se sont jamais préoccupés de sonder les mystères de la création au sein de laquelle ils vivent ${ }^{16}$. " L'infériorité des connaissances, les croyances et les superstitions expliqueraient la situation précaire des Africains.

Malgré la publication, par le pape Pie XI, de l'encyclique Rerum Ecclesice en 1926, dans laquelle il rappelle l'importance pour les missionnaires de ne pas considérer les peuples étrangers comme " inférieurs ${ }^{17}$, l'Afrique est régulièrement décrite par les missionnaires canadiens-français comme une terre à coloniser sur le plan culturel et spirituel. Dans le premier numéro de la revue Visite annuelle de Notre-Dame d'Afrique aux foyers canadiens en 1908, les Sœurs blanches évoquent l'œuvre de "régénération morale " aux fondements de leur action, principalement auprès de la femme noire ou musulmane en Afrique $^{18}$. Certes, le discours des Sours blanches ne reflète pas

16 Odilon Chevrier, o.m.i., "La science des Basutos ", Le Devoir, 16 juin 1927, p. 7.

17 Pie XI, Rerum Ecclesia, paragraphe 26, sur le site Web du Vatican, [http://www. vatican.va/holy_father/pius_xi/encyclicals/documents/hf_p-xi_enc_28021926_ rerum-ecclesiae_en.html].

18 "L’œuvre de la régénération de la femme musulmane en Afrique ", Visite annuelle de Notre-Dame d'Afrique aux foyers canadiens, vol. 1, no 1 (1908), p. 9-12. 
totalement un rapport de domination univoque. Par exemple, une sœur trouve les Africains "intelligents " et "même instruits ${ }^{19}$ ", ce qui contraste avec le discours de certains collègues. Néanmoins, règle générale, le langage utilisé masque à peine le rapport hiérarchique entre colonisateur et colonisé. Plusieurs missionnaires usent des qualificatifs « primitif » et " arriéré » pour caractériser l'état des peuples africains. L'autre est appréhendé à partir de sa propre perspective, de sa culture et de ses connaissances.

En général, la connaissance de l'Afrique réfère à des conceptions vagues qui trahissent certains préjugés : l'inconnu, l'exotisme, la pauvreté, la maladie, l'islam et, quelques fois, le cannibalisme ou la sorcellerie sont évoqués par des missionnaires ${ }^{20}$. Par leur côté énigmatique, ces représentations frappent davantage l'imaginaire collectif que les récits plus descriptifs et anthropologiques de certains missionnaires. Les références au colonialisme sont presque nulles à cette époque. Somme toute, cette période demeure une phase d'organisation du missionnariat. Au tournant des années 1930, plus de mille missionnaires canadiens-français sont désormais actifs à travers le monde.

\section{La colonisation et l'expansion des missions (1929-1948)}

La Grande Dépression, la crise des années 1930 et la Deuxième Guerre mondiale ont des conséquences sur le missionnariat canadienfrançais. On assiste à un ralentissement de la croissance des missions africaines. Les revues missionnaires de l'époque font état d'un manque flagrant de ressources matérielles et pécuniaires. Les revenus des principales œuvres pontificales missionnaires n'ont pas la

19 Sour Marie Coecilia, «Lettre de M. Coecilia à la révérende mère supérieure du postulat de Québec ", Visite annuelle de Notre-Dame d'Afrique aux foyers canadiens, vol. $1, \mathrm{n}^{\circ} 7$ (juillet 1912), p. 158-159.

${ }^{20}$ Ce type de références linguistiques est fréquent dans les récits quotidiens publiés dans les revues missionnaires. Par exemple, G. Bertrand des p.b., «Les jumeaux au Buruli ", Missions d'Afrique des Pères blancs, vol. 18, nº 3 (mars 1922), p. 80-88; Père Eugène Déry, "Lettre du r.p. Eugène Déry ", Missions d'Afrique des Pères blancs, vol. 18, nº 12 (décembre 1922), p. 358-364. 
croissance escomptée : il leur faut vingt ans avant de recueillir des revenus annuels similaires à ceux de $1929^{21}$. Les congrégations réussissent néanmoins à se développer faiblement, particulièrement grâce aux effectifs missionnaires qui continuent de croître ${ }^{22}$.

La fin de la Deuxième Guerre mondiale amène une nouvelle division des colonies. Le paganisme, l'islam et l'athéisme deviennent des ennemis pouvant nuire non seulement au développement des missions, mais aussi à la progression personnelle des missionnés. Le " problème qui hante notre esprit [...] peut-être le plus grand problème actuel, dans le domaine missionnaire [...] l'Afrique centrale sera-t-elle chrétienne, musulmane ou athée ${ }^{23}$ ? " demande l'évêque hollandais Joseph Blomjous, responsable des Pères blancs d'Afrique.

Le continent africain représente, aux yeux des catholiques, une terre fertile à l'expansion du catholicisme. Les possibilités de conversion et d'évangélisation se comptent par milliers, voire par millions. Au Mozambique, le père blanc Joseph Laliberté affirme que « les conversions sont nombreuses et relativement faciles. Les gens font preuve de bonnes dispositions. À noter que ces peuplades sont extrêmement polies et hospitalières. Toutefois il arrive qu'un certain nombre résistent à l'appel du Maître ${ }^{24} »$. Cette résistance est attribuée aux superstitions et aux rites païens des Africains, et notamment des « sorciers qui ont longtemps causé de l'embêtement " aux missionnaires ${ }^{25}$.

21 Éric Desautels, «Le missionnariat canadien-français : d'un engagement religieux à une action coopérative déconfessionnalisée ", communication présentée à la $31^{e}$ Conférence de la Société internationale de sociologie des religions, Aix-enProvence (France), $1^{\text {er }}$ juillet 2011.

22 De 1932 à 1949, le nombre de missionnaires passe de 1595 à 3133 (Adrien Bouffard, «L'Église missionnaire canadienne », Messages, vol. 15, n ${ }^{\text {os }} 1-2$ (janvierjuin 1959), p. 1).

${ }_{23} \mathrm{M}^{\mathrm{gr}} \mathrm{J}$ Joseph Blomjous, p.b., "Le problème africain ", Annales de la Propagation de la Foi, vol. 24, no 3 (mai-juin 1947), p. 63. Des textes de Blomjous sont fréquemment publiés dans les revues missionnaires.

${ }^{24}$ André Roy, "Le R.P. Joseph Laliberté, p.b. au Nyassa (Afrique) ", Annales de la Propagation de la Foi, vol. 22, no 2 (mars-avril 1945), p. 40.

25 Ibid. 
Au Canada français, les missionnaires profitent de la Semaine missionnaire et des expositions missionnaires ${ }^{26}$ afin de décrire différentes facettes de la culture africaine. Des témoignages, des photographies et des objets sont exposés. À l'occasion du tricentenaire de Montréal, l'exposition Ville-Marie missionnaire, organisée à l'Oratoire Saint-Joseph en septembre 1942, attire près de 250000 personnes $^{27}$. Si elles soulignent la contribution des missionnaires à l'ouverture du Québec sur le monde, ces expositions concrétisent également l'écart culturel qui sépare l'Occident de l'Orient.

Ce clivage culturel ne constitue pas nécessairement un problème puisque l'objectif consiste à " civiliser » les populations non chrétiennes qui accusent un retard sur les " porteurs » de la civilisation occidentale et chrétienne que représentent les missionnaires ${ }^{28}$. En 1931, le directeur de la Société de Missions-Étrangères, le chanoine J.-Avila Roch, soutient que la conversion des païens constitue " l'œuvre essentielle " de sa société et qu'il faut combattre les préjugés des infidèles « au moyen des ouvres de charité corporelle et spirituelle ${ }^{29}$ ». Il considère que la culture chrétienne occidentale est supérieure à celle des Africains puisqu'elle transmet le message du Christ dans une forme « moderne » et « civilisée ». Seuls les missionnaires seraient

${ }^{26}$ La première exposition missionnaire est tenue à Joliette en juillet 1927. Le Devoir et La Patrie ont d'ailleurs amplement couvert l'événement tous les jours. Voir aussi le compte rendu détaillé publié en 1931 sur la Semaine missionnaire et l'exposition missionnaire organisées à Montréal. Il s'agissait du deuxième événement du genre en raison du succès de Joliette ( $\mathrm{M}^{\mathrm{gr}}$ Alphonse Deschamps, La semaine missionnaire de Montréal, Montréal, Librairie Beauchemin, 1930). Voici la liste des expositions missionnaires tenues au Canada français : Joliette (1927), Montréal (1930 et 1943), Ottawa (1935), Trois-Rivières (1935), Saint-Hyacinthe (1937), Sherbrooke (1941). Concernant le tricentenaire de la ville de Montréal, l'exposition Ville-Marie missionnaire en 1942 a aussi connu un vif succès.

27 Secrétariat du comité missionnaire, Ville-Marie missionnaire : 1642-1942, Montréal, Comité missionnaire, 1943, p. 221.

28 Par exemple, sur la question des missionnaires comme porteurs de civilisation, voir Gérard Paquet, o.m.i., "Des prêtres noirs! ", Annales de la Propagation de la Foi, vol. 6, no 4 (août 1929), p. 153.

29 Deschamps, La semaine missionnaire de Montréal, p. 244 et 245. 
capables d'inculquer aux indigènes une discipline efficace, à savoir " la morale de la religion chrétienne ${ }^{30}$ ". Cette transmission à partir d'une morale chrétienne serait même recherchée par les familles africaines : "Ils cherchent à leur faire donner une éducation pratique, intellectuelle, morale, adaptée à leur temps et à leur milieu ${ }^{31}$. " Demeurant sensiblement le même qu'à la période précédente, le discours missionnaire fait état de thèmes telles la sorcellerie, la polygamie, l'alimentation parfois curieuse, la faune de la brousse africaine ainsi que la simplicité de leur vie ${ }^{32}$.

Toutefois, à partir des années 1940, plusieurs missionnaires usent d'une interprétation davantage anthropologique et tentent de comprendre ou, du moins, de décrire les rites et les croyances des Africains ${ }^{33}$. Par exemple, le père Joseph Laliberté soutient que les "Noirs sont encore au stade patriarcal " et que leurs coutumes sont fascinantes sur le plan sociologique ${ }^{34}$. Cette ouverture à la compréhension des autres cultures s'amorce plus lentement pour les prêtres et le clergé missionnaires, qui semblent conserver une pensée plus traditionnelle et dogmatique par comparaison aux frères et aux sœurs missionnaires ${ }^{35}$.

30 La Direction, "Missions noires, moissons d'or ", Annales de la Propagation de la Foi, vol. 24, n 1 (janvier-février 1947), p. 4.

31 "Après le jardin d'enfants... l'École ménagère ", Visite de Notre-Dame d'Afrique aux foyers canadiens, vol. 25, $\mathrm{n}^{\circ} 4$ (avril 1936), p. 109.

32 Encore une fois, les récits quotidiens des missionnaires évoquent fréquemment de tels thèmes. Voir Sœur Marie-de-Pontmain, « Nos frères les lépreux : bienheureux ceux qui pleurent ", Visite de Notre-Dame d'Afrique aux foyers canadiens, vol. 23, no 8 (août 1936), p. 232-235; "Vieux métiers en faillite ", Missions d'Afrique des Pères blancs, vol. 29, n 7 (juillet 1933), p. 203-208; P. Arnoux, p.b., " La lumière chasse les ténèbres ", Missions d'Afrique des Pères blancs, vol. 29, n 6 (juin 1933), p. 169-174.

33 Par exemple, Paul-Émile Vachon, p.b., "Obsèques royales ", Annales de la Propagation de la Foi, vol. 22, no 6 (novembre-décembre 1945), p. 146-147 et 150. Voir aussi plusieurs écrits des Sœurs blanches.

${ }^{34}$ André Roy, "Le R.P. Joseph Laliberté, des p.b. et les Missions de la Côte d'Or ", Annales de la Propagation de la Foi, vol. 22, n 2 (mars-avril 1945), p. 40.

35 La proximité des frères et sœurs avec les missionnés est une piste de réflexion intéressante. Cette proximité leur permettrait non seulement de mieux comprendre 
Les deux premières phases du missionnariat canadien-français prolongent la domination coloniale occidentale en Afrique : la domination des missionnaires ne dépare pas la domination des marchands et des militaires. Les missionnaires canadiens-français ne se positionnent pas par rapport à ce colonialisme, mais on sent qu'ils privilégient une forme d'autonomie en proposant de former des communautés ou un clergé indigène fort plutôt que de se référer aux nations colonisatrices ${ }^{36}$. On évoque les paroles des hauts dirigeants de l'Église catholique qui proposent de convertir le semblable par le semblable : "La nécessité du clergé indigène s'impose aujourd'hui plus que jamais. C'est là qu'est la clef pour résoudre le difficile problème missionnaire, le secret pour accélérer d'un rythme précipité les conquêtes évangéliques aujourd'hui si promettantes $[\text { sic }]^{37}$. »

\section{Les mouvements nationalistes et la décolonisation (1948-1958)}

La fin de la Deuxième Guerre mondiale, la création de l'Organisation des Nations Unies le 26 juin 1945 et la ratification de la Déclaration universelle des droits de l'homme en décembre 1948 annoncent de profonds changements sur la scène internationale. Le climat devient propice aux luttes d'indépendance et affecte les fondements de la vocation missionnaire. Les années 1950 et 1960 concordent avec

la perspective de l'autre, mais aussi d'être mieux placés dans l'espace missionnaire pour créer un espace d'innovation et d'échanges culturels. Une sœur blanche, tout en affirmant que ses collègues doivent enseigner les vérités de la foi, croit que « la Sœur a toute liberté, et elle en profite, pour faire des observations générales et même particulières, des admonestations s'il y a nécessité » (Sœur M.-Saint-Patrice, "La persévérance des femmes chrétiennes au Ruanda ", Visite de Notre-Dame d'Afrique aux foyers canadiens, vol. 25, n 10 (octobre 1935), p. 301).

36 J.-B. Durand, "Appel d'un missionnaire en faveur d'une communauté de Sœurs indigènes ", Annales de la Propagation de la Foi, vol. 23, no 1 (janvier-février 1946), p. 18-19; Jean-Paul Pelletier, "Entrevue avec le r.p. Louis-Joseph Cabana, p.b. », Annales de la Propagation de la Foi, vol. 24, nº 2 (mars-avril 1947), p. 35.

37 Citation de $\mathrm{M}^{\mathrm{gr}}$ Salotti, président de l'CEuvre de Saint-Pierre-Apôtre (J.-M. Quirion, o.m.i., "Un problème! Une solution! ", Annales de la Propagation de la Foi, vol. 23, no 1 (janvier-février 1946), p. 24). 
" un processus de passation des pouvoirs des missionnaires aux Églises locales » qui va de pair avec la décolonisation ${ }^{38}$ et qui modifie le regard sur les Africains et l'Afrique. Par l'intermédiaire d'encycliques publiées entre 1948 et 1958, c'est d'abord au sein du discours papal que s'amorce ce changement ${ }^{39}$.

L'émergence d'un nouvel encadrement des droits collectifs et individuels sur la scène internationale contribue à transformer graduellement la représentation de l'Afrique. Dans un éditorial daté de 1950, la revue Annales de la Propagation de la Foi évoque l'importance de lutter contre les préjugés et le paganisme afin d'œuvrer au « relèvement » de la femme noire africaine, c'est-à-dire à l'amélioration de son état social et de ses droits fondamentaux ${ }^{40}$. Dans un article relatant le $50^{\mathrm{e}}$ anniversaire de la fondation de la congrégation des Pères blancs au Canada, le père Joseph Larochelle souligne le progrès accompli par rapport à l'image de l'Afrique originellement projetée : c'est la fin des récits sur les "mangeurs d'hommes ", les empoisonnements et les coups de lance et de flèche reçus par les missionnaires ${ }^{41}$. On retrouve des articles décrivant la réalité africaine dans les journaux canadiens-français, notamment une chronique hebdomadaire de Pères blancs, dans L'Action catholique, qui porte sur différentes facettes de la vie quotidienne (culture, faune, flore, art africain, histoire du continent, martyrs) ${ }^{42}$.

38 Jean-François Zorn, "Engagements politiques des acteurs de la mission après 1945? Tendances méthodologiques et thématiques ", dans Caroline Sappia et Olivier Servais (dir.), Missions et engagement politique après 1945 : Afrique, Amérique latine, Europe, Paris, Karthala, 2010, p. 13-15.

39 Dans Evangelii Praecones, en 1951, le pape Pie XII affirme, par exemple, la nécessité de modifier les fondements mêmes de la mission face aux changements sociaux mondiaux amorcés depuis Rerum Ecclesia.

${ }^{40}$ La Direction, "L'Église et le relèvement de la femme noire ", Annales de la Propagation de la Foi, vol. 27, n 3 (mai-juin 1950), p. 58-61.

${ }^{41}$ Joseph Larochelle, p.b., "Un bond! », Missions d'Afrique des Pères blancs, vol. 47, no 7-8 (juillet-août 1951), p. 195-196.

42 Par exemple, Un Père Blanc, "Mort d'un vétéran ", L’Action catholique, 23 janvier 1955, p. 22; Un Père Blanc, "Faune africaine ", L'Action catholique, 3 février 1957, p. 22-23; Un Père Blanc, "Un bienheureux martyr ", L’Action catholique, 30 janvier 1955, p. 22. 
Pourtant, des préoccupations similaires à celles des deux périodes précédentes persistent. Des dirigeants de mission dénoncent le paganisme et l'islam, qui représentent une menace ${ }^{43}$ :

Le nord est païen et musulman tandis que l'on peut dire que le sud est païen et chrétien. Le vieux musulman du nord [du Nigéria] repose sur sa vieille culture, le jeune chrétien a des ambitions illimitées. Dans les deux cas les païens sont très arriérés, mais ceux du sud se rende $[s i c]$ compte de leur état et veulent en sortir [...] Maintenant, lorsque vous allez mettre cet appât de l'école sous le nez d'une tribu amorphe, peu consciente de son infériorité et peu soucieuse d'améliorer sa situation, vous avez une séance de pêche à la carpe avec hameçon ${ }^{44}$.

L'analogie de la pêche à la carpe rappelle que le changement et l'innovation dans le champ missionnaire sont plutôt lents. On observe la continuation de l'objectif de conversion et d'évangélisation ainsi que des relents de prosélytisme ${ }^{45}$. Les Africains sont présentés différemment lorsqu'ils se sont convertis, ce qui tend à confirmer l'hypothèse d'Edward Said à propos du regard de l'autre : les Africains sont représentés plus positivement lorsqu'ils adhèrent à la religion et à la culture chrétienne occidentale.

43 Pour la relation des missionnaires au paganisme et à l'athéisme, voir Gaston Mathieu, p.b., "Quel sera le sort de l'Afrique?", Annales de la Propagation de la Foi, vol. 33, n 6 (novembre-décembre 1956), p. 157-159 et 166; Sœur MarieJean-Chrysostome, "À travers l'Afrique centrale ", Saurs blanches, vol. 40, n 1 (janvier 1951), p. 82-85; Eugène, p.b., "Les catéchistes ", L’Action catholique, 5 septembre 1954, p. 22. Pour l'islam, voir notamment, La Direction, "Qui l'emportera? Le Christ ou Mahomet ", Annales de la Propagation de la Foi, vol. 26, n 5 (septembre-octobre 1949), p. 115-117; La Direction, «Les missions d'Afrique aux confins de l'Islam ", Annales de la Propagation de la Foi, vol. 33, $\mathrm{n}^{\circ} 2$ (mars-avril 1956), p. 29-31.

44 Rodrigue Roberge, c.s.sp., "Problème missionnaire ", Annales de la Propagation de la Foi, vol. 26, nº 6 (novembre-décembre 1949), p. 163.

45 Ce qui est aussi constaté dans d'autres revues missionnaires de l'époque : Catherine Foisy, "Oralité dans Le Précurseur et Missions Étrangères (1945-1962) : entre prosélytisme et visée pédagogique ", Études d'histoire religieuse, vol. 76 (2010), p. 57-69. 
Aux yeux des dirigeants de mission, une réelle compréhension réciproque entre l'Afrique et l'Occident ne pourra être atteinte que par la formation d'un clergé indigène, comme l'affirme le supérieur des Pères blancs en Afrique, $\mathrm{M}^{\mathrm{gr}}$ Louis Durrieu : «Le clergé indigène est traité sur un pied d'égalité avec les prêtres étrangers et a déjà commencé à assumer la parfaite direction des églises fondées ${ }^{46}$. " À partir du début des années 1950, le laïcat indigène devient de plus en plus un incontournable pour bâtir l'Église catholique africaine ${ }^{47}$. L'effet de la religion catholique sur les autres cultures se fait ressentir. Il faut s'accommoder aux contextes locaux et nationaux ainsi que mieux comprendre les cultures et croyances d'autrui : les missionnaires sont maintenant plus nuancés et "s'adaptent " progressivement aux décolonisations. Certaines communautés de frères et de sœurs s'ajustent mieux aux réalités et aux besoins concrets des missionnés : les frères reçoivent parfois plus d'estime que les pères de l'aveu même d'un père blanc ${ }^{48}$.

Il ne s'agit plus d'imposer sa culture ou sa civilisation, mais plutôt de miser sur la notion d' " adaptation ". Il faut désormais "s'adapter non pour dominer, mais pour donner et recevoir. Pour donner en tenant compte de ce que les autochtones attendent de nous [...] [c'est-à-dire] le témoignage d'une vie vraiment évangélique ", affirme la sœur blanche Marie-Vincent Ferrier ${ }^{49}$. Aussi les Sœurs blanches maintiennent-elles un discours d'ouverture et de proximité aux autres

46 Jean-Paul Pelletier, "Les Pères blancs en Afrique noire ", Annales de la Propagation de la Foi, vol. 27, n ${ }^{\circ} 5$ (septembre-octobre 1950), p. 138. Voir aussi «Les Missions des Pères Servites de Marie ", Annales de la Propagation de la Foi, vol. 33, n 6 (novembre-décembre 1956), p. 166.

47 Le père mariste Jacques Pelletier évoque ce besoin criant aux Îles Salomon en 1955. Même s'il se situe en dehors de l'Afrique, il relate une tendance intéressante en période d'après-guerre, c'est-à-dire le besoin de se libérer, sur le terrain missionnaire, des frères, sœurs, prêtres et laïcs étrangers : «Laïcs en pays de missions ", Annales de la Propagation de la Foi, vol. 32, no 5 (septembre-octobre 1955), p. 130-131.

48 Père A. Prost, p.b., " Pour aider les missionnaires, des auxiliaires indispensables ", L'Action catholique, 20 octobre 1957, p. 22.

49 Sœur M.-Vincent Ferrier, "Adaptation missionnaire ", Annales de la Propagation de la Foi, vol. 33, no 1 (janvier-février 1956), p. 9. 
cultures et religions. Elles veulent respecter et comprendre pour mieux s'adapter : "Il ne s'agit pas seulement de découvrir une mentalité différente, dans un cadre de vie analogue au nôtre; il s'agit du problème de l'interpénétration de deux civilisations ${ }^{50}$. " L'influence d'une " théologie de l'adaptation ", qui a émergé dans des cercles catholiques de l'entre-deux-guerres avant de s'imposer dans les années 1950, selon l'historien Claude Prudhomme, est perceptible dans le discours missionnaire ${ }^{51}$.

Sur le terrain, des missionnaires explorent d'autres voies comme la coopération et le service social ${ }^{52}$. Ces nouvelles formes d'engagement ou d'organisation se révèlent intéressantes puisqu'elles délaissent partiellement l'objectif de stricte conversion des païens. Il s'agit maintenant de spécialiser sa vocation dans certains domaines afin d'éduquer les missionnés, de développer l'économie locale, de créer des réseaux d'entraide, etc. : les domaines des services sociaux, des coopératives, de la santé, de l'agriculture et de l'éducation sont maintenant privilégiés. À partir du milieu des années 1950, il ne s'agit plus exclusivement de convertir et de prêcher, mais de bâtir des

50 "Sœurs noires", Sæurs blanches, vol. 42, n 1 (janvier-février 1953), p. 8.

51 Claude Prudhomme, "La mission au $\mathrm{xx}^{\mathrm{e}}$ siècle : triomphe, crise et mutations du côté catholique, en particulier à travers l'exemple des Spiritains ", dans Coulon (dir.), Action française, décolonisation, $M^{g r}$ Lefebvre, p. 13.

52 Se référer à des articles sur le service social, les coopératives et l'éducation populaire qui se multiplient sur le terrain missionnaire africain : La Direction, "Le laïcat missionnaire ", Annales de la Propagation de la Foi, vol. 25, n 3 (mai-juin 1948), p. 57-60; Sœur Gérin-Lajoie, "Le Service Social en Mission ", Annales de la Propagation de la Foi, vol. 32, no 3 (mai-juin 1955), p. 66; Georges-Henri Lévesque, o.p., "Apostolat missionnaire et formation sociale ", Annales de la Propagation de la Foi, vol. 32, n 2 (mars-avril 1955), p. 55; Sœur M.-Vincent Ferrier, "Adaptation missionnaire ", Annales de la Propagation de la Foi, vol. 33, $\mathrm{n}^{\circ} 1$ (janvier-février 1956), p. 9. Une intéressante conférence prononcée par l'abbé Conrad Côté, p.m.e., présente, en utilisant théoriquement le cas philippin, l'apport du service social pour les missions canadiennes-françaises, notamment en vue d'implanter une action et une justice sociales plus efficaces : «Le Service Social tel qu'on pourrait l'appliquer aux Philippines ", Annales de la Propagation de la Foi, vol. 32, no 1 (janvier-février 1955), p. 3-5. 
communautés chrétiennes. Pour les missionnaires, si les Africains sont libres de leur corps avec la fin de l'esclavage, le temps est venu de "libérer les esprits par le moyen des écoles ${ }^{53}$ ". Selon eux, l'éducation catholique libérerait les esprits dans la foulée des mouvements africains d'émancipation.

\section{Le tournant des années 1950 en ce qui concerne les mouvements nationalistes}

Contrairement à d'autres parties du globe ${ }^{54}$, les terres africaines semblent relativement bien accepter la présence de religieux. Les missionnaires canadiens-français ont à se positionner par rapport aux luttes politiques et nationales. On craint que l'émancipation se produise au profit du communisme et de l'athéisme. C'est vers 1955, dans un contexte concordant avec la guerre d'Algérie, que le discours missionnaire clarifie sa position face aux mouvements indépendantistes et nationalistes africains. Avec la multiplication des mouvements de contestation, les missionnaires se dissocient davantage des pays colonisateurs même s'ils évoquent très peu l'impérialisme et le colonialisme. À cet effet, une sœur blanche rappelle le modèle qu'a représenté $\mathrm{M}^{\text {gr }}$ Lavigerie, le fondateur des congrégations des Sœurs blanches et des Pères blancs, qui avait heurté " les principes de colonisation alors en vigueur " en enseignant à des autochtones et des colons de manière simultanée dès la fin du $\mathrm{XIX}^{\mathrm{e}}$ siècle $^{55}$. Ces propos réfèrent du même coup à la " position stratégique " des missionnaires canadiens-français :

53 Père Roberge, p.s.-e.m., "Rien ne sert de prêcher... il faut bâtir! ", Annales de la Propagation de la Foi, vol. 29, nº 3 (mai-juin 1952), p. 68. Sur le rôle des écoles catholiques, voir aussi René Dufresne, p.b., "Visite des écoles ", L'Action catholique, 13 mai 1956, p. 22-23.

54 Le cas de la Chine où les missionnaires ont été martyrisés ou expulsés est un très bon exemple. Voir l'ouvrage d'Alvyn J. Austin, Saving China: Canadian Missionaries in the Middle Kingdom, 1888-1959, Toronto, University of Toronto Press, 1989.

55 Sœur Marie-Andrée-du-Sacré-Cour, "Les Instituts missionnaires africains ", Sours blanches, vol. 41, nº 2 (février 1952), p. 37. 
Ces derniers avaient l'avantage de se situer un peu en dehors des luttes politiques qui affectaient les relations entre les nations européennes. Le fait qu'ils pouvaient jouer sur leur appartenance aux deux cultures française et anglaise leur permettait d'opérer aussi bien dans les colonies britanniques que françaises. Les Canadiens français étaient ainsi acceptés dans des régions du continent où la présence d'autres missionnaires aurait paru suspecte ${ }^{56}$.

Le discours missionnaire est, à l'évidence, loin d'être un bloc monolithique en ce qui concerne les mouvements nationalistes et indépendantistes africains dans les années 1950. Un regard ambivalent prend forme dans la crainte de voir l'islam, le communisme et l'athéisme gagner du terrain en pleine guerre froide. Les missionnaires adoptent ainsi une position paradoxale, à la fois en faveur des émancipations nationales africaines et réfractaires à celles-ci. Ce regard ambivalent n'est pas étranger aux réflexions développées au Canada français dès l'entre-deux-guerres en ce qui concerne le séparatisme : la doctrine sociale de l'Église ne permet pas de se soustraire de la domination politique étrangère, sauf « en cas d'abus de pouvoir des autorités constituées ${ }^{57}$ ".

D’une part, les missionnaires semblent parfois réticents puisque ces mouvements peuvent se concrétiser dans la violence. Les mouvements nationalistes ne doivent pas non plus se réaliser au profit d'un matérialisme athée associé à l'empire soviétique. La compétition est farouche : «[...] la propagande de nos adversaires tente de soutenir aux yeux des Nord-Africains, surtout qu'une religion comme la religion catholique, dont le siège est à Rome, constitue une religion européenne dont on se sert pour essayer de maintenir le colonialisme ${ }^{58}$.

56 Jean-Philippe Warren, "Les commencements de la coopération internationale Canada-Afrique : le rôle des missionnaires canadiens ", dans Jean-Bruno Mukanya Kaninda-Muana (dir.), Les relations entre le Canada, le Québec et l'Afrique depuis 1960, Paris, L'Harmattan, 2010, p. 30.

57 Jean-Claude Dupuis, "Nationalisme, séparatisme et catholicisme dans l'entredeux-guerres ", dans Michel Sarra-Bournet (dir.), Les nationalismes au Québec du XIX $X^{e}$ au XXI siècle, Québec, Les Presses de l'Université Laval, 2001, p. 116.

58 La Direction, "Les écoles catholiques de l'Afrique ", Annales de la Propagation de la Foi, vol. 33, nº 6 (novembre-décembre 1956), p. 143. 
Selon des dirigeants de mission, la pensée colonisatrice et civilisatrice reposerait davantage sur une fausse interprétation. Cette dernière serait plutôt alimentée par la propagande de leurs adversaires idéologiques. Les missionnaires se distancient progressivement, entre 1948 et 1958, d'une pensée coloniale et d'une perspective strictement occidentale de la religion catholique en Afrique.

D'autre part, on retrouve des missionnaires ouverts à une émancipation des esprits autant individuelle que collective. Or, selon eux, c'est le catholicisme qui serait le mieux placé pour produire cette lumière sur le continent africain. Toute révolution ou tout mouvement nationaliste basé sur le paganisme ou le communisme ne serait point bénéfique. Il faut que l'objectif traditionnel d'évangélisation des païens continue à s'accomplir sous une nouvelle forme, ce qui sera l’apanage de la période d'apogée.

\section{La période de mutation et d'apogée (1958-1968)}

Les années 1950 ont révélé un passage progressif vers un plus grand intérêt pour les enjeux de la décolonisation. L'historien français Claude Prudhomme expose lui aussi ce passage chez les missions chrétiennes et souligne qu'à partir des années 1960 un " nouveau positionnement, fruit de la logique missionnaire et du pragmatisme, s'impose et traduit la volonté de tourner la page sur la colonisation ${ }^{59}$ ". Une telle période transitoire est présente au sein des missions catholiques agissant dans le tiers-monde. Le chercheur Charles-Édouard Harang soutient que la période de 1957-1967 correspond à "l'axe central du mouvement de décolonisation, d'émergence du tiersmonde et de leur réception au sein du catholicisme français ${ }^{60}$ ". Cette période traduit le passage entre colonisation et renouveau au sein de l'espace missionnaire. Un dirigeant de mission se défend de faire partie des colonisateurs en soutane : "Il n’y a pas de colonialisme

\footnotetext{
59 Claude Prudhomme, "Mission, colonisation, décolonisation : vue d'ensemble ", dans Borne et Falaize (dir.), Religions et colonisation, p. 71.

${ }^{60}$ Harang, Quand les jeunes catholiques découvrent le monde, p. 25.
} 
dans l'Église. C'est l'égalité des classes et des hommes devant Dieu dans la distribution de la justice pour tous ${ }^{61}$. "

Publiée en 1957, l'encyclique Fidei Donum du pape Pie XII devient la référence pour guider l'esprit missionnaire et réorienter le rôle des prêtres missionnaires. Elle consacre un transfert de pouvoir vers les Églises locales ${ }^{62}$, un changement de position qui se reflète rapidement dans le discours missionnaire :

Or, n'apparaît-il pas de toute nécessité, à cette heure où les peuplades naguère les plus primitifs $[s i c]$ réclament l'émancipation, quand ils ne l'arrachent pas par la violence à leurs anciens maitres, n'apparait-il pas de la plus élémentaire prudence pour garantir la conservation, sinon l'accroissement des positions acquises par le catholicisme, en ce pays, de leur donner des chefs religieux de leur propre esprit ${ }^{63}$ ?

Le clergé autochtone obtient ainsi plus d'autonomie : «Les communautés et les patries prennent de plus en plus conscience d'ellesmêmes; elles se constituent et affirment chaque jour davantage leur personnalité. Nous ne pouvons ni ne voulons rester indifférents ou passifs devant cette évolution si chargée d'inconnues, mais aussi d'espérances ${ }^{64}$. "Selon ces évêques, la liberté et la dignité chrétiennes seraient cruciales afin de vivre pleinement les mouvements de libération, ce qui ferait défaut au marxisme et au communisme ${ }^{65}$.

61 "Dans les œuvres sociales ", Annales de la Propagation de la Foi, vol. 38, n 5 (septembre-octobre 1961), p. 135.

${ }^{62}$ Voir l'enthousiasme de certains missionnaires face à ce transfert de pouvoir : F.-X. Lapointe, p.b., "Un grand jour pour l'Église du Nyasaland ", L'Action catholique, 5 juin 1960, p. 22 ; M ${ }^{\mathrm{gr}}$ J.-D. Desrosiers, "L'Église au Basutoland ", Annales de la Propagation de la Foi, vol. 36, n 6 (novembre-décembre 1959), p. 144-148.

63 Abbé Honorius Provost, "L'œuvre pontificale de Saint-Pierre-Apôtre », Annales de la Propagation de la Foi, vol. 37, nº 6 (novembre-décembre 1960), p. 156.

${ }^{64}$ Lettre de onze archevêques de l'Afrique française, "Les problèmes de l'Église en Afrique française ", Annales de la Propagation de la Foi, vol. 35, n 4 (juillet-août 1958), p. 86.

${ }^{65}$ Les missionnaires s'attaquent au communisme et au marxisme qui représentent une solution crédible au catholicisme social afin de contrer ces inégalités à travers la " mauvaise répartition des biens à l'intérieur et sur le communisme " 
Dans l'esprit de Fidei Donum, un oblat missionnaire rappelle d'ailleurs que la formation d'un clergé indigène fort représentait la priorité dès l'arrivée en terres africaines :

parce que les autochtones sont mieux qualifiés que les autres pour adapter le christianisme à la mentalité et aux besoins de leurs compatriotes et l'intégrer ainsi de façon stable dans la vie et la civilisation nationales; mais surtout parce que le clergé indigène est une nécessité qui découle de la nature même de l’Église ${ }^{66}$.

Voilà un changement important dans le discours des prêtres et du clergé missionnaires. Les Africains sont désormais vus comme aptes à prendre en mains leur destinée et à développer et former l'Église catholique. Le catholicisme aurait ainsi la capacité de s'adapter aux contextes culturels et sociaux. Certaines idées au cœur du concile Vatican II se manifestent à différents niveaux ecclésiaux et de manière plus uniforme comparativement aux périodes précédentes : adaptation des méthodes missionnaires aux " réalités modernes ${ }^{67}$ ", accent mis sur le laïcat dans le cadre de l'activité apostolique ${ }^{68}$ et démocratisation des institutions.

Désirant insuffler un esprit missionnaire, le concile Vatican II provoque une réflexion au sein du missionnariat canadien-français. Comme on l'évoquait souvent à l'époque, il y a un besoin d'" adaptation " au monde moderne et aux mouvements nationalistes. La constitution d'une Église universelle passe d'abord et avant tout par les Églises locales, avec leur contexte social et culturel particulier. C'est ainsi que doit être envisagé l'aggiornamento de l'Église dans le tiers-monde. Le concile contribue à façonner l'image d'une Afrique

(La Direction, "Des événements qui doivent nous faire réfléchir », Annales de la Propagation de la Foi, vol. 42, no 1 (janvier-février 1965), p. 6).

${ }^{66}$ Desrosiers, "L'Église au Basutoland », p. 147.

${ }^{67} \mathrm{M}^{\mathrm{gr}} \mathrm{N}$.-A. Labrie, "Concile et esprit missionnaire ", Annales de la Propagation de la Foi, vol. 42, n 5 (septembre-octobre 1965), p. 114; M ${ }^{\mathrm{gr}}$ N.-A. Labrie, "Le concile", Annales de la Propagation de la Foi, vol. 43, n 2 (mars-avril 1966), p. 34.

${ }_{68}$ Par exemple, ce numéro consacré aux laïcs : Saurs blanches, vol. 51, n 2 (marsavril 1962), p. 2-24; Desrosiers, "L'Église au Basutoland », p. 148. 
jeune et dynamique dont la vitalité se traduit, entre autres, par son " influence morale et spirituelle ${ }^{69}$ ». L'adaptation n'est pas seulement matérielle et institutionnelle; elle doit aussi se réaliser dans les esprits. Les échanges interreligieux sont valorisés dans le discours missionnaire ${ }^{70}$ et les missions se tournent vers le "service social ", le dialogue avec les non-chrétiens et la collaboration avec la population ${ }^{71}$.

Les missionnaires reconnaissent aussi l'apport du concile dans la relation aux autres races, cultures et religions : " Le Concile a examiné à la lumière de l'Évangile le problème de la discrimination raciale, politique, sociale ${ }^{72}$." L'attitude des missionnaires envers les autres cultures et religions change de plus en plus. En revanche, certains missionnaires croient toujours que le paganisme causerait, par ses croyances et ses traditions, un état d'infériorité chez les femmes africaines $^{73}$. Le catholicisme constituerait encore une fois le moyen à privilégier pour accéder à davantage de droits et de libertés, ce qui laisse toujours entrevoir un rapport inégalitaire entre cultures.

En ce qui concerne les mouvements nationalistes, la revue des Sœurs blanches rappelle que "le réveil de l'Afrique signifie une volonté de près de deux cents millions de Noirs d'affirmer leur participation active, consciente, et d'apporter leur contribution originale à l'organi-

${ }^{69}$ Roger Tessier, p.b., "Cette fois... Toute l'Afrique sera au rendez-vous! », Annales de la Propagation de la Foi, vol. 40, n 2 (mars-avril 1963), p. 38. Voir aussi les articles subséquents de Roger Tessier sur le concile et l'Afrique dans les revues Annales de la Propagation de la Foi et Missions d'Afrique des Pères blancs.

${ }^{70}$ L'espace missionnaire a toujours constitué un espace d'innovation et de rencontres interculturelles des plus intéressants au sein de l'Église catholique. Toutefois, ces échanges dans le champ missionnaire deviennent centraux, au tournant des années 1960, dans les réflexions du missionnariat.

${ }^{71}$ Cardinal Zougrana, "Problèmes missionnaires d'aujourd'hui ", Annales de la Propagation de la Foi, vol. 43, no 3 (juillet-août 1966), p. 90-91.

72 Robert Lepire, ptre, "Continuer le concile ", Feuillet missionnaire de l'Union pontificale missionnaire, vol. III, $\mathrm{n}^{\circ} 5$ (mai 1968).

73 Jean-Paul Pelletier, "L'Église dans la société africaine ", Annales de la Propagation de la Foi, vol. 36, n 2 (mars-avril 1959), p. 34; Sœur Marie de Saint-Honoré, "Impression d'arrivée ", Annales de la Propagation de la Foi, vol. 35, nº 3 (maijuin 1958), p. 63-65 et 75 . 
sation réfléchie de la planète au même titre que tous les autres hommes $^{74}$ ». Sur l'indépendance du Congo, les Sœurs blanches citent un article congolais à la une de leur revue dans lequel on trouve une opinion tranchée : "Le peuple congolais a droit à son indépendance au même titre que les autres peuples du globe. C'est un droit fondamental, naturel, sacré qu'aucune doctrine ne peut lui disputer, aucune puissance lui arracher [...] [Il faut] l'indépendance effective dans l'interdépendance des nations libres, la libération totale du Congo de toute forme de tutelle ${ }^{75}$. "Les Sœurs blanches reprennent même des citations de W.E. B. Dubois, évoquant le problème de la ségrégation raciale et la crainte des Africains de la domination patronale occidentale ${ }^{76}$.

Des tensions persistent cependant face aux mouvements nationalistes dans certains pays, comme au Congo où les missionnaires canadiens-français constatent des lacunes dans les droits et libertés : "À Bukavu, tous les droits de l'homme sont foulés aux pieds. On est livré à la plus arbitraire façon d'agir sans recours possible à la justice. Plus de liberté ni d'expression, ni de presse, ni de conviction ${ }^{77}$. " Ces mouvements sont vus plus négativement lorsqu'ils limitent la liberté d'expression, de conviction et d'action des catholiques sur le terrain. De son côté, l'évêque congolais Augustin Awaka rappelle aux missionnaires canadiens-français que les Congolais perçoivent la modération de certains missionnaires face aux mouvements nationalistes comme une opposition à leur indépendance et à leur émancipation. Les missionnaires sont alors mis sur un pied d'égalité avec

74 Jacques Rabemananjara, «Les Noirs à travers le monde », Sours blanches, vol. 48, $\mathrm{n}^{\circ}$ 3-4 (mars-avril 1959), p. 6.

75 L. de Wasseige, "Indépendance, mot magique », Scurs blanches, vol. 48, n 7-8 (juillet-août 1959), p. 3.

76 Marguerite Dhellemmes, "Tradition, évolution, et rôle de la mère ", Socurs blanches, vol. 51, n 1 (janvier-février 1962), p. 4-6. Voir aussi ce numéro, Soeurs blanches, vol. 48, no 2 (mars-avril 1959), p. 2-24.

77 La Direction, "L'Église catholique au Kivu ", Annales de la Propagation de la Foi, vol. 38, n 2 (mars-avril 1961), p. 43. 
les Belges et les laïcs, soutient-il ${ }^{78}$. Le quotidien L'Action catholique rapporte d'ailleurs des articles de Congolais qui lancent des appels pour que les missionnaires puissent continuer leur travail en éducation, en santé et en service social malgré la haine et les violences qui sévissent au cours des luttes pour l'indépendance ${ }^{79}$. Bref, il y a reconnaissance de la légitimité du nationalisme par les missionnaires lorsqu'il est possible de concilier la religion catholique et les indépendances nationales : «car loin de bouder nationalisme et indépendance nationale, l'Église les reconnaît légitimes ${ }^{80}$. " Encore une fois, ces mouvements ne doivent cependant pas se concrétiser dans la violence.

Avec ces luttes, la représentation sociale de l'Afrique poursuit sa mutation. Au début des années 1960, l'abbé Lionel Groulx évoque cette transformation en cours. L'exotisme et l'espérance d'un El Dorado laissent plutôt place à une vision axée sur la pauvreté et la misère : "Cette pauvreté relative, peut-être plus exagérée que réelle, n’empêche pas cette portion du globe de prendre l'aspect d'un "monde nouveau" [...] Bref, pour sortir de son état de pays sous-développé, il ne manquerait à l'Afrique qu'une plus forte injection de capitaux et de techniques améliorées ${ }^{81}$. " Cette analyse de Groulx cadre bien avec le discours véhiculé par les missionnaires, qui se recentrent sur la question de la pauvreté, de la souffrance et de la misère en Afrique.

$\mathrm{Si}$ le concile Vatican II place ce continent et l'activité missionnaire apostolique au cœur de ses prérogatives, les missionnaires cherchent à combattre la misère et la pauvreté africaine par la charité, l'entraide et la coopération ${ }^{82}$. La Révolution tranquille amorcée, les questions

78 L'abbé Augustin Awaka, «Regards sur l'Église au Congo », Annales de la Propagation de la Foi, vol. 39, n 1 (janvier-février 1962), p. 9.

79 Temps Nouveaux, "Les événements du Congo belge ", L'Action catholique, 4 septembre 1960, p. 22 ; Temps Nouveaux, "Appel aux élites congolaises », L'Action catholique, 11 septembre 1960, p. 22.

${ }^{80}$ J.-P., "Un bon Dieu noir si possible... ", Missions d'Afrique des Pères blancs, vol. 56, n 11 (décembre 1960), p. 324-326.

${ }^{81}$ Lionel Groulx, Le Canada français missionnaire : une autre grande aventure, Montréal, Éditions Fides, 1962, p. 253-254.

${ }^{82}$ Voir notamment J.-M. Chamberland, "Ah! Si j'avais des sous!... ", Annales de la Propagation de la Foi, vol. 39, n 4 (juillet-août 1962), p. 100-101. 
de la charité, de l'amour de son prochain et du développement du tiers-monde deviennent omniprésentes dans le discours véhiculé au Canada français par la presse et les intellectuels catholiques. La représentation de l'Afrique transmise dans la sphère publique par les missionnaires évolue également. Les reportages télévisés sur le missionnariat deviennent plus fréquents et permettent à la population canadienne-française de mieux comprendre les enjeux locaux et culturels en Afrique ${ }^{83}$. Ces reportages évoquent la misère humaine et l'état de pauvreté dans le champ apostolique missionnaire. C'est là un des défis des missionnaires des années 1960 : au moment des indépendances africaines, il faut combattre les inégalités sociales émergentes.

Les pays «sous-développés » représentent dorénavant une priorité pour les missionnaires ${ }^{84}:$ il faut contribuer socialement, matériellement et spirituellement à ces populations. Les missions civilisatrices laissent place à des missions de coopération, de service social ou d'entraide humanitaire. Concrétisant cette mutation institutionnelle, la création de l'Organisation catholique canadienne pour le développement et la paix en 1967 est inspirée par les principes de "l'Action catholique spécialisée ainsi que par l'“option préférentielle pour les pauvres” définies au Concile Vatican II et à la Conférence des Évêques d'Amérique latine à Medellin en $1968^{85}$ ». Une nouvelle ère s'ouvre sur

${ }^{83}$ Par exemple, Société Radio-Canada, Les Archives de Radio-Canada, "Les Pères blancs au Ghana ", La Bonne Nouvelle, 14 janvier 1962, 25 min 48 s, [En ligne], [http://archives.radio-canada.ca/societe/religion_spiritualite/clips/6271/] ; Idem, "Des missionnaires canadiens au Japon ", La Bonne Nouvelle, 27 novembre 1960, $11 \mathrm{~min} 34 \mathrm{~s}$, [En ligne], [http://archives.radio-canada.ca/societe/religion_ spiritualite/clips/6269/] ; Idem, "Le cardinal Léger de retour d'Afrique ", La Bonne Nouvelle, 2 février 1964, [En ligne], [http://archives.radio-canada.ca/societe/ religion_spiritualite/clips/6291/].

${ }^{84}$ Le décret $A d$ Gentes et l'encyclique Populorum Progressio amènent la question du développement au cœur du discours missionnaire.

${ }^{85}$ Catherine LeGrand, "L'axe missionnaire catholique entre le Québec et l'Amérique latine : une exploration préliminaire ", Globe : revue internationale d'études québécoises, vol. 12, $\mathrm{n}^{\circ} 1$ (2009), p. 43-66. 
l'activité missionnaire et sur la représentation de l'Afrique véhiculée dans le discours catholique canadien-français.

\section{Une nouvelle représentation de l'Afrique et une transformation des missions}

Entre les années 1900 et 1968, l'évolution du discours missionnaire révèle une transformation du rapport à l'autre et de la représentation sociale de l'Afrique. Les tensions issues des mouvements de colonisation et de décolonisation ne sont pas étrangères à cette mutation. Au cours des périodes d'organisation et d'expansion des missions canadiennes-françaises, le discours demeure essentiellement ancré dans l'idéologie de l'orientalisme. L'Afrique demeure surtout représentée à travers des archétypes référant à l'exotisme, à l'inconnu et à l'aventure dans la brousse africaine. Il fallait alors combattre le paganisme et les croyances " précaires " des Africains. Les fondements de l'activité missionnaire tournant principalement autour d'une mission civilisatrice de conversion, la compréhension et le respect des autres cultures sont limités. Les missionnaires jugent l'état de l'Afrique et de ses populations du point de vue de leur propre culture.

La Deuxième Guerre mondiale et le développement d'une sphère internationale amènent une éthique mondiale nouvelle qui inspire les organisations et les acteurs internationaux tout autant que les congrégations missionnaires. Cette période transitoire vers une nouvelle représentation de l'Afrique est aussi marquée par l'émergence d'une " théologie de l'adaptation " qui devient prédominante dans le discours missionnaire. Les missionnaires s'adaptent ainsi aux contextes locaux et nationaux pour mieux s'insérer dans les communautés chrétiennes africaines. On assiste alors au passage, chez les missionnaires, de la transmission d'une morale chrétienne à la transmission de valeurs universelles relevant davantage d'une éthique laïque. La fondation d'organisations non gouvernementales laïques dans les années 1960 transforme aussi le champ d'action missionnaire : un modèle coopératif et déconfessionnalisé entre en compétition avec 
l'action missionnaire catholique traditionnelle. De plus, la vocation missionnaire se spécialise et se professionnalise progressivement.

Le discours transmis dans la presse et les quotidiens canadiensfrançais évolue dans le temps. La Révolution tranquille donne un nouvel élan aux relations internationales du Canada et du Québec. L'État québécois devient plus actif et des médias comme la télévision et la presse augmentent l'intérêt pour les pays du tiers-monde. Une nouvelle représentation de l'Afrique prend forme. Au Québec, le modèle de missionnariat que représente $\mathrm{M}^{\mathrm{gr}}$ Paul-Émile Léger frappe l'imaginaire québécois. Léger quitte son poste de cardinal pour se consacrer au tiers-monde, aux plus démunis et aux lépreux. La représentation du missionné devient associée à l'image du pauvre et du malade qu'il faut secourir au nom de la charité chrétienne. Cette dévotion envers les plus démunis ouvre la voie à une forme renouvelée d'engagement dans le tiers-monde qui sera caractéristique de la deuxième moitié $\mathrm{du} \mathrm{xx}^{\mathrm{e}}$ siècle : un engagement social relevant du modèle coopératif et de l'entraide internationale, plus détaché des institutions et de la morale catholiques. 